\title{
CLINICAL VALIDATION OF THE CE-IVD MARKED THERASCREEN MGMT KIT IN A COHORT OF GLIOBLASTOMA PATIENTS.
}

Véronique Quillien ${ }^{1,2}$, Audrey Lavenu ${ }^{3,4}$, François Ducray, ${ }^{5,6}$, David Meyronet, ${ }^{5,6}$, Olivier Chinot $^{7}$, Frédéric Fina ${ }^{8}$, Marc Sanson ${ }^{9}$, Catherine Carpentier ${ }^{9}$ 'Lucie Karayan-Tapon ${ }^{10,11}$, Pierre Rivet ${ }^{11}$, Natacha Entz-Werle ${ }^{12,13}$, Michèle Legrain ${ }^{12}$, Emmanuèle Lechapt Zalcman ${ }^{14}$, Guenaelle Levallet ${ }^{14}$, Fabienne Escande ${ }^{15}$, Carole Ramirez ${ }^{15}$, Dan Chiforeanu ${ }^{16}$, Elodie Vauleon ${ }^{1,2}$ and Dominique FigarellaBranger ${ }^{7,17}$.

\section{Authors' affiliation :}

1. Centre Eugène Marquis, F-35042 Rennes, France

2. INSERM U1242, Université de Rennes 1, F-35043 Rennes, France

3. Université Rennes 1, Faculté de Médecine, F-35043 Rennes, France

4. INSERM CIC 0203, Université de Rennes 1, F-35043 Rennes, France

5. Hospices Civils de Lyon, F- 69394, Lyon, Cedex, France

6. Université de Lyon1, F-69622 Villeurbanne, France

7. CHU Timone, F-13385 Marseille, France

8. Faculté de médecine secteur Nord, F-13916 Marseille, France

9. Sorbonne Universités UPMC Université Paris 06, INSERM CNRS, U1127, UMR 7225, ICM, F75013 Paris, France

10. INSERM U1084, Université de Poitiers, F-86021 Poitiers, France

11. CHU de Poitiers, F-86021 Poitiers, France

12. CHRU Hautepierre, F67098 Strasbourg, France

13. EA 3430, Progression tumorale et microenvironnement. Approches translationnelles et épidémiologie. Université de Strasbourg, F-67000 Strasbourg, France

14. CHU Caen, Département de pathologie, F-14000 Caen, France

15. CHRU de Lille, F-59037 Lille, France.

16. CHU de Rennes, F-35000 Rennes, France

17. INSERM U911 CRO2, Université de la Méditerranée, F-13385 Marseille, France

Corresponding author: Véronique Quillien, Centre Eugène Marquis, F-35042 Rennes, France. Tel. No. (33)299253190, fax no. (33)299253164. Email address: v.quillien@rennes.unicancer.fr 


\section{ABSTRACT}

BACKGROUND, pyrosequencing is recognized as a strong technique to analyze the MGMT status of glioblastoma patients. The most commonly used assay, quantifies the methylation levels of CpGs 74 to 78. A more recent CE-marked In Vitro Diagnostic Medical Device (CE-IVD) assay, Therascreen, analyzes CpGs 76-79.

METHODS, we performed a comparison of these two assays to evaluate the potential impact of this shift in analyzed CpGs. Therascreen analysis was centrally performed for 102 glioblastoma patients, who were part of a prospective multicenter trial.

RESULTS, a strong correlation was observed for the mean values of the 4 or 5 analyzed CpGs, with lower values recorded using the Therascreen assay, especially for values greater than $20 \%$. When considering a classification in 3 categories (>12\%: methylated; $\leq 8 \%$ : unmethylated; $9-12 \%$ : grey zone), $93 \%$ of patients were identically classified between the two assays. Using a binary classification, $95 \%$ and $97 \%$ of patients were identically classified with cut-offs of $8 \%$ and $12 \%$, respectively. A strong prognostic significance was observed for both assays: median overall survival were 15.9 months and 34.9 months for respectively unmethylated and methylated patients with either test.

CONCLUSION, the results demonstrate that these assays may be used interchangeably.

Key words: Glioblastoma, MGMT methylation, Pyrosequencing, CE-IVD kit 


\section{INTRODUCTION}

Qiagen currently sells numerous assays for quantitative measurements of MGMT (methylguanine methyltransferase) methylation status using the pyrosequencing technique. Hs_MGMT_01_PM PyroMark CpG assay (ref PM 00149702) analyzes 7 CpGs located upstream of DMR1 and DMR2 regions, where methylation has significantly been correlated with expression [1]. This assay must therefore be avoided for a clinical purpose. Two additional assays (ref 970032 and 972032) quantify the methylation levels of CpGs 74 to 78 . They are respectively optimized for use with the PyroMark Q24 and Q96. These two similar assays are currently the most widely used and validated pyrosequencing assays [2]. They will be further referred to as PSQ. In 2011, a fourth assay was launched: the Therascreen MGMT Pyro Kit (Thera). This assay, in contrast to the others, is a CEmarked In Vitro Diagnostic Medical Device (CE-IVD) that meets all requirements of the EC Directive 98/79.

Several countries around the world have adopted the international standard ISO 15189, which specifies requirements for quality and competence in medical laboratories. Among the preferred procedures described in this international standard are those specified in the instructions for use of in vitro medical devices. Furthermore, as the steps of validation into the laboratory are less extensive for CE-IVD assays, laboratories tend to favor this type of assay. Thera appears to be a suitable choice for MGMT testing and some studies have reported strong analytical performances for this assay [3, 4]. However, this assay quantifies the methylation levels of CpGs 76 to 79 instead of CpGs 74 to 78 . One of the strengths of PSQ for MGMT testing relies on the several independent studies that are concordant with the threshold levels that discriminate glioblastoma (GBM) patients as being good or poor responders to Temozolomide (TMZ) treatment [2]. As a heterogeneous pattern of methylation can be observed for some tumors, the shift in the CpGs analyzed between the two assays could potentially impact the result (= average methylation percentage of the tested $\mathrm{CpGs}$ ). It is therefore mandatory to validate the cut-off for the Thera assay.

We have recently performed a prospective dedicated multicenter trial, which allowed us to validate the use of PSQ in a daily practice. For the present study, we analyzed 102 frozen GBM patients from this 
trial with the Thera assay and compared the results to those obtained previously with the standard PSQ test.

\section{MATERIALS and METHODS}

\section{Patients and samples}

Samples were analyzed from patients enrolled in a prospective study dedicated to the validation of two techniques to assess MGMT status [5]. Patients were enrolled for this study between the dates of March 11, 2009 and June 29, 2011 from 8 French centers. Eligible patients had histologically confirmed de novo-glioblastoma, between the ages of 18-70 and presented with no contraindications, as dictated by the Stupp protocol. The protocol was approved by the Rennes medical ethics committee and informed consent was obtained from each patient.

DNA was extracted from 3 primary cell lines (RNS85/96 and 175), which were used as quality controls in each series of tests.

\section{MGMT promoter methylation analysis}

DNA extractions from frozen clinical samples and sodium bisulfite treatment were performed at each center according to local procedures. Samples with a histologically estimated tumor cell content below $40 \%$ were excluded from the study. The Thera test was centrally performed on any remaining bisulfite treated DNA following completion of the main portion of the project. Thera was performed using the Therascreen MGMT Pyro Kit (ref. 971061, Qiagen, France) according to the manufacturer's instructions. The average percentage of the $4 \mathrm{CpGs}$ tested was considered to calculate the cut-off.

\section{Statistical analysis}

Statistical analysis was performed using $R$ statistical software (version 2.13.0, http://www.Rproject.org). The function risksetAUC (package risksetROC) in the R statistical software was used to obtain the area under the ROC curve. Additionally, the Harrell's C index [6] was calculated using the validate function (in Design package). To study OS and PFS, cumulative event curves (censored endpoints) were established using the Kaplan-Meier method. 


\section{RESULTS}

\section{Study population}

Among the 112 samples of patients initially analyzed with PSQ, 102 were available for the Thera analysis. The median Progression Free Survival (PFS) for these 102 patients was 9.5 months (8.8 $11.2 ; 95 \% \mathrm{Cl})$ and the median Overall survival (OS) was 20.6 months $(18.7-23.0 ; 95 \% \mathrm{Cl})$.

\section{Analysis of intra-laboratory reproducibility of Thera}

Each control was evaluated in 6 different series. The mean values were 4\% for RNS85 (range: 4-4\%), 16\% for RNS175 (range: $15-17 \%$ ) and 33\% (range: $32-34 \%$ ) for RNS96. The reproducibility CVs were $5 \%$ for RNS175 and 3\% for RNS96. All the results were identical for RNS85, which is close to the limits of quantification previously published for pyrosequencing (4\%).

\section{Comparison of MGMT methylation results obtained with the $\mathbf{2}$ kits}

The median percentages of methylation for the studied population were $7 \%$ when using Thera (range $1-65 \%$ ) and $8 \%$ when using PSQ (range 1-84\%) (Table 1). A strong correlation was observed for CpG76, 77, 78 and CpG mean analyzed using the PSQ and Thera assays. However, almost systematically, lower results were recorded with Thera, especially for values greater than $20 \%$ (Figure 1).

\section{Validation of the pre-defined cut-offs $8 \%$ and $12 \%$ for Thera}

We recently recommended a classification of MGMT promoter methylation status into three subgroups: "unmethylated" (0-8\%), "methylated" (13-100\%) and a grey zone for patients with intermediate values $(9-12 \%)$ [5]. The cut-offs 8 and $12 \%$ were tested in this series of patients. The percentages of patients classified as "methylated", when employing a cut-off of $8 \%$ and $12 \%$ for PSQ, were $50 \%$ and $44 \%$ with AUCROC values of 0.69 for OS (Table 2). These data are almost identical to those obtained for the overall population ( $n=112$ patients, 49\%, 44\% and AUCROC values of 0.69 and 0.70), indicating the absence of bias in the selection of the 102 patients for the present cohort. The 
percentages of patients classified as "methylated" when employing a cut-off of $8 \%$ and $12 \%$ for Thera were $45 \%$ and $41 \%$, with AUCROC values of 0.68 for OS (Table 2). With a classification in 3 categories (methylated/unmethylated/grey zone), $93 \%$ of patients were identically classified using the two assays. With a binary classification (methylated/unmethylated), $95 \%$ and $97 \%$ of patients were identically classified with cut-offs of $8 \%$ and $12 \%$ respectively (Table 3 ). Table 4 shows the pyrosequencing results, as well as the time to death and time to progression for the patients differently classified by the two assays.

Kaplan-Meier survival curves displaying the OS of patients dichotomized according to these cut-offs are presented in Figure 2. At a methylation cut-off of $8 \%$, median OS were 34.7 months $(95 \% \mathrm{Cl} 23.0-$ 39.4) versus 15.9 months $(95 \% \mathrm{Cl} 13.2-19.0)$ for respectively methylated and unmethylated patients analysed with Thera, with a p value of $1.310^{-7}$. Results were 30.0 months ( $\left.95 \% \mathrm{Cl} 22.9-39.1\right)$ versus 15.9 months $(95 \% \mathrm{Cl} 13.1-19.0)$ with a $\mathrm{p}$ value of $2.610^{-9}$ for PSQ. At a methylation cut-off of $12 \%$, results were 34.9 months $(95 \% \mathrm{Cl} 24.5-43.8)$ versus 17.0 months $(95 \% \mathrm{Cl} 13.7-19.5)$ with a $\mathrm{p}$ value of $3.210^{-7}$ for Thera and 34.9 months $(95 \% \mathrm{Cl} 24.5-40.8)$ versus 16.4 months $(95 \% \mathrm{Cl} 13.7-19.1)$ with a $\mathrm{p}$ value of $1.410^{-8}$ for PSQ. However, only few samples had methylation between $9-12 \%$ and it was not possible to individualize them in our study.

\section{DISCUSSION}

MGMT promoter methylation is recognized as an effective predictor of response to TMZ for newly diagnosed GBM patients. Among the different techniques to analyze MGMT status, pyrosequencing is regarded as a very robust technique and its clinical utility has been validated in several independent studies [7-14]. Pyrosequencing provides the percentage of methylated alleles of each CpG site analyzed and generally the average of the different sites is used to classify patients as "methylated" or "unmethylated". The 5 most commonly analyzed CpGs are CpGs 74 to 78 and commercial kits are available to assess them, allowing reproducible and comparable results from one laboratory to another. The more recently launched Thera kit quantifies the methylation levels of CpGs 76 to 79 . We have previously described that methylation can be heterogeneous from one site to the other [15]; analyzing different CpGs could therefore have an impact on the mean result. As a corollary the thresholds optimized for a combination of CpGs may not be optimal for additional combinations. 
A comparison of $\mathrm{CpGs}$ one by one demonstrated a strong correlation between the results obtained with PSQ and Thera for common CpGs 76, 77 and 78, but with lower results with Thera for values above $15-20 \%$. In our own experience, bisulfite treated DNA is very stable, so the delay of 2-3 months between Thera analysis and bisulfite treatment can't explain these lower results. The same observation was performed for the mean values. As we propose threshold values of 8 and $12 \%$, this difference has a minor impact for patient's classification as "methylated" and "unmethylated". With cutoffs at $12 \%$ and $8 \%, 97 \%$ and $95 \%$ of patients were identically classified according to the two techniques. When considering a three-class classification, among the 7 patients differently classified, 6 would have been classified as methylated with one technique and as being in the grey zone with the alternate technique. Specific authors have proposed higher cut-offs between $25 \%$ and $35 \%$ to classify patients $[8,11,16]$. In our study, the higher the cut-off increased, the higher the percentage of discordant cases. For example, values of $25 \%, 35 \%$ and $50 \%$ associated with $6 \%, 9 \%$ and $21 \%$ of patients having been differently classified within the two techniques.

To validate a clinical cut-off, we tested values of 8 and $12 \% .41 \%$ of patients had values $>12 \%$ with Thera compared to $44 \%$ with PSQ. $55 \%$ of patients had values $\leq 8 \%$ with Thera compared to $50 \%$ with PSQ. In all discordant cases except one, result were between $9 \%$ and $12 \%$ with one assay and $>12 \%$ or $\leq 8 \%$ with the other assay. A very good prognostic significance was observed for both assays and both cut-offs. The reduced risk of death in the case of results above $12 \%$ was slightly higher with PSQ compared to Thera (HR: $0.27, p<1.00 \mathrm{E}^{-06}$ versus HR: $0.30, \mathrm{p}=1.00 \mathrm{E}^{-06}$ ), as we had already reported using a non-company producted PSQ test [15]. For this study, we have not been able to establish the prognosis of patients whose tumors present with percentages of methylation comprise between $9 \%$ and $12 \%$. Since it is for this category of patients that we observed the higher number of discordant cases between the two techniques, we recommend, as for PSQ, to consider patients with a mean methylation percentage $\leq 8 \%$ as unmethylated, those with a mean methylation percentage $>12 \%$ as methylated and those with percentages of methylation between $9 \%$ and $12 \%$ as being in a grey zone. An additional study analyzing a series of GBM FFPE samples with Thera found that the optimal cut-off value to dichotomize patients was $\geq 8 \%$ [17], providing confidence in our choice of thresholds.

In conclusion, Thera and PSQ may both be used to analyze MGMT status in glioblastomas. Intralaboratory reproducibility for Thera was good and others have previously reported a high analytical 
performance of this kit, including inter-laboratory reproducibility [3]. The same cut-offs can be applied for the two kits, although they do not interrogate exactly identical CpGs.

\section{ACKNOWLEDGEMENTS}

We gratefully thank M. Marty for her technical contribution. The authors acknowledge the Centre de Ressources Biologiques (CRB) Santé of Rennes (BB-0033-00056), the tumorothèque de Caen Basse Normandie and the AP-HM tumor bank or Marseille (AC 2013-1786) for managing patient samples. Funding was provided by the French Ministry of Health (Support for Costly Cancer Technical Evaluation - STIC - Gov-0478)

INFORMED CONSENT: the experiments were undertaken with the understanding and written consent of each subject. The study was conformed with the code of ethics of the word medical association. 


\section{References}

[1] D.S. Malley, R.A. Hamoudi, S. Kocialkowski, D.M. Pearson, V.P. Collins and K. Ichimura, A distinct region of the MGMT CPG island critical for transcriptional regulation is preferentially methylated in glioblastoma cells and xenografts, Acta Neuropathol 121 (2011), 651-61.

[2] M. Bienkowski, A.S. Berghoff, C. Marosi, A. Wohrer, H. Heinzl, J.A. Hainfellner and M. Preusser, Clinical Neuropathology practice guide 5-2015: MGMT methylation pyrosequencing in glioblastoma: unresolved issues and open questions, Clin Neuropathol 34 (2015), 250-7.

[3] M. Preusser, A.S. Berghoff, C. Manzl, M. Filipits, A. Weinhausel, W. Pulverer, K. Dieckmann, G. Widhalm, A. Wohrer, E. Knosp, C. Marosi and J.A. Hainfellner, Clinical Neuropathology practice news 1-2014: pyrosequencing meets clinical and analytical performance criteria for routine testing of MGMT promoter methylation status in glioblastoma, Clin Neuropathol 33 (2014), 6-14.

[4] A. Luquain, S. Magnin, D. Guenat, J.L. Pretet, G. Viennet, S. Valmary-Degano and C. Mougin, [Analysis of MGMT methylation with the therascreen((R)) MGMT Pyro((R)) Kit (Qiagen). A method verification], Ann Biol Clin (Paris) 73 (2015), 665-70.

[5] V. Quillien, A. Lavenu, F. Ducray, M.O. Joly, O. Chinot, F. Fina, M. Sanson, C. Carpentier, L. Karayan-Tapon, P. Rivet, N. Entz-Werle, M. Legrain, E.L. Zalcman, G. Levallet, F. Escande, C. Ramirez, D. Chiforeanu, E. Vauleon and D. Figarella-Branger, Validation of the high-performance of pyrosequencing for clinical MGMT testing on a cohort of glioblastoma patients from a prospective dedicated multicentric trial, Oncotarget 7 (2016), 61916-61929.

[6] F.E. Harrell, Jr., K.L. Lee and D.B. Mark, Multivariable prognostic models: issues in developing models, evaluating assumptions and adequacy, and measuring and reducing errors, Stat Med $\mathbf{1 5}$ (1996), 361-87.

[7] V. Quillien, A. Lavenu, L. Karayan-Tapon, C. Carpentier, M. Labussiere, T. Lesimple, O. Chinot, M. Wager, J. Honnorat, S. Saikali, F. Fina, M. Sanson and D. Figarella-Branger, Comparative assessment of 5 methods (methylation-specific polymerase chain reaction, MethyLight, pyrosequencing, methylation-sensitive high-resolution melting, and immunohistochemistry) to analyze 06methylguanine-DNA-methyltranferase in a series of 100 glioblastoma patients, Cancer 118 (2012), 4201-11.

[8] J. Dunn, A. Baborie, F. Alam, K. Joyce, M. Moxham, R. Sibson, D. Crooks, D. Husband, A. Shenoy, A. Brodbelt, H. Wong, T. Liloglou, B. Haylock and C. Walker, Extent of MGMT promoter methylation correlates with outcome in glioblastomas given temozolomide and radiotherapy, Br J Cancer 101 (2009), 124-31.

[9] A. Christians, C. Hartmann, A. Benner, J. Meyer, A. von Deimling, M. Weller, W. Wick and M. Weiler, Prognostic value of three different methods of MGMT promoter methylation analysis in a prospective trial on newly diagnosed glioblastoma, PLoS One 7 (2012), e33449. 
[10] A.B. Havik, P. Brandal, H. Honne, H.S. Dahlback, D. Scheie, M. Hektoen, T.R. Meling, E. Helseth, S. Heim, R.A. Lothe and G.E. Lind, MGMT promoter methylation in gliomas-assessment by pyrosequencing and quantitative methylation-specific PCR, J Transl Med 10 (2012), 36.

[11] G. Reifenberger, B. Hentschel, J. Felsberg, G. Schackert, M. Simon, O. Schnell, M. Westphal, W. Wick, T. Pietsch, M. Loeffler and M. Weller, Predictive impact of MGMT promoter methylation in glioblastoma of the elderly, Int J Cancer 131 (2012), 1342-1350.

[12] V.P. Collins, K. Ichimura, Y. Di, D. Pearson, R. Chan, L.C. Thompson, R. Gabe, M. Brada and S.P. Stenning, Prognostic and predictive markers in recurrent high grade glioma; results from the BR12 randomised trial, Acta Neuropathol Commun 2 (2014), 68.

[13] D. Shen, T. Liu, Q. Lin, X. Lu, Q. Wang, F. Lin and W. Mao, MGMT promoter methylation correlates with an overall survival benefit in Chinese high-grade glioblastoma patients treated with radiotherapy and alkylating agent-based chemotherapy: a single-institution study, PLoS One 9 (2014), e107558.

[14] L. Lattanzio, M. Borgognone, C. Mocellini, F. Giordano, E. Favata, G. Fasano, D. Vivenza, M. Monteverde, F. Tonissi, A. Ghiglia, C. Fillini, C. Bernucci, M. Merlano and C. Lo Nigro, MGMT promoter methylation and glioblastoma: a comparison of analytical methods and of tumor specimens, Int J Biol Markers 30 (2015), e208-16.

[15] V. Quillien, A. Lavenu, M. Sanson, M. Legrain, P. Dubus, L. Karayan-Tapon, J. Mosser, K. Ichimura and D. Figarella-Branger, Outcome-based determination of optimal pyrosequencing assay for MGMT methylation detection in glioblastoma patients, J Neurooncol 116 (2014), 487-96.

[16] G. Brigliadori, F. Foca, M. Dall'Agata, C. Rengucci, E. Melegari, S. Cerasoli, D. Amadori, D. Calistri and M. Faedi, Defining the cutoff value of MGMT gene promoter methylation and its predictive capacity in glioblastoma, J Neurooncol 128 (2016), 333-9.

[17] C.Y. Hsu, H.L. Ho, S.C. Lin, Y.C. Chang-Chien, M.H. Chen, S.P. Hsu, Y.S. Yen, W.Y. Guo and D.M. Ho, Prognosis of glioblastoma with faint MGMT methylation-specific PCR product, J Neurooncol 122 (2015), 179-88. 


\begin{tabular}{|l|c|c|c|c|}
\hline & Mean & Median & Min & Max \\
\hline Thera1 (CPG76) & 16 & 4 & 0 & 90 \\
\hline Thera2 (CPG77) & 16 & 6 & 0 & 66 \\
\hline Thera3 (CPG78) & 13 & 6 & 1 & 67 \\
\hline Thera4 (CPG79) & 20 & 10 & 1 & 89 \\
\hline Thera mean & 16 & 7 & 1 & 65 \\
\hline
\end{tabular}

\begin{tabular}{|l|c|c|c|c|}
\hline & Mean & Median & Min & Max \\
\hline PSQ1 (CPG74) & 23 & 7 & 0 & 86 \\
\hline PSQ2 (CPG75) & 25 & 9 & 1 & 85 \\
\hline PSQ3 (CPG76) & 22 & 8 & 1 & 85 \\
\hline PSQ4 (CPG77) & 20 & 7 & 0 & 87 \\
\hline PSQ5 (CPG78) & 17 & 7 & 1 & 86 \\
\hline PSQ mean & 21 & 8 & 1 & 84 \\
\hline
\end{tabular}

Table 1: Mean, Median and extreme values for each $\mathrm{CpG}$ and for the mean of the 4 or $5 \mathrm{CpGs}$ analyzed with the PSQ and Thera assays. 


\begin{tabular}{|l|l|l|l|l|l|}
\hline Variable & Cut-off $(\%)$ & $\mathrm{HR}$ & $\mathrm{p}$ & AUCROC & CHarrel \\
\hline THERA & 8 & 0.30 & $1,00 \mathrm{E}^{-06}$ & 0.68 & 0.69 \\
\hline THERA & 12 & 0.30 & $1,00 \mathrm{E}^{-06}$ & 0.68 & 0.68 \\
\hline PSQ & 8 & 0.25 & $<1,00 \mathrm{E}^{-06}$ & 0.69 & 0.69 \\
\hline PSQ & 12 & 0.27 & $<1,00 \mathrm{E}^{-06}$ & 0.69 & 0.69 \\
\hline
\end{tabular}

Table 2: comparison of the prognostic impacts when evaluating MGMT promoter methylation with therascreen pyrosequensing (Thera) and "standard" pyrosequencing (PSQ). The previously determined cut-offs of $8 \%$ and $12 \%$ were tested to determine the associated Hazard ratio (HR) and the level of significance (represented by the $p$ value, which is to compare to $1.3 / 1000$ with the multiple comparison correction of Bonferroni), after adjustment on age and Karnofsky score. The prediction errors were globally evaluated and reported as the Area Under the ROC Curve (AUCROC) and the Harrell's C index. 


\begin{tabular}{l|c|c|c|}
\hline \multicolumn{1}{l}{$\begin{array}{c}\text { PSQ } \\
\text { Thera }\end{array}$} & $\leq 8$ & $9-12$ & $>12$ \\
\cline { 2 - 4 } $9-12$ & 51 & 4 & 1 \\
\cline { 2 - 4 }$>12$ & 0 & 2 & 2 \\
\hline & 0 & 0 & 42 \\
\hline
\end{tabular}

B

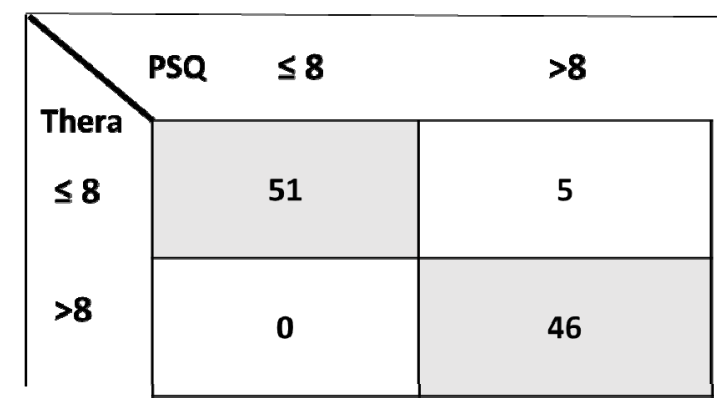

C

\begin{tabular}{|c|c|c|}
\hline & $\leq 12$ & $>12$ \\
\hline$\leq 12$ & 57 & 3 \\
\hline$>12$ & 0 & 42 \\
\hline
\end{tabular}


Table 3. Agreement between the two assays. Number of patients identically classified dichotomizing patients in 3 classes with cut-offs at 8 and $12 \%$ (A) or in two classes with cut-offs at $8 \%$ (B) or $12 \%$ (C).

\begin{tabular}{|c|c|c|c|c|c|c|c|c|c|c|}
\hline $\begin{array}{l}\text { Patient's } \\
\text { number }\end{array}$ & & PSQ1 & PSQ2 & $\begin{array}{r}\text { PSQ3 } \\
\text { Thera1 } \\
\end{array}$ & $\begin{array}{r}\text { PSQ4 } \\
\text { Thera2 } \\
\end{array}$ & $\begin{array}{r}\text { PSQ5 } \\
\text { Thera3 }\end{array}$ & Thera4 & $\begin{array}{l}\text { PSQ mean } \\
\text { Thera mean }\end{array}$ & $\begin{array}{l}\text { Time to } \\
\text { recurrence } \\
\text { (months) }\end{array}$ & $\begin{array}{c}\text { Time to } \\
\text { death } \\
\text { (months) }\end{array}$ \\
\hline \multirow[b]{2}{*}{1017} & PSQ & 10 & 34 & 12 & 14 & 4 & & 15 & \multirow[b]{2}{*}{181} & \multirow[b]{2}{*}{642} \\
\hline & Thera & & & 10 & 12 & 4 & 12 & 10 & & \\
\hline \multirow[b]{2}{*}{2154} & PSQ & 9 & 11 & 9 & 8 & 8 & & 9 & \multirow[b]{2}{*}{594} & \multirow[b]{2}{*}{1256} \\
\hline & Thera & & & 11 & 8 & 4 & 10 & 8 & & \\
\hline \multirow[b]{2}{*}{7056} & PSQ & 9 & 9 & 9 & 7 & 10 & & 9 & \multirow[b]{2}{*}{84} & \multirow[b]{2}{*}{84} \\
\hline & Thera & & & 5 & 4 & 7 & 10 & 7 & & \\
\hline \multirow[b]{2}{*}{2022} & PSQ & 17 & 9 & 9 & 11 & 6 & & 10 & \multirow[b]{2}{*}{279} & \multirow[b]{2}{*}{402} \\
\hline & Thera & & & 4 & 7 & 6 & 11 & 7 & & \\
\hline \multirow[b]{2}{*}{8004} & PSQ & 6 & 16 & 17 & 7 & 7 & & 11 & \multirow[b]{2}{*}{454} & \multirow[b]{2}{*}{821} \\
\hline & Thera & & & 13 & 6 & 5 & 8 & 8 & & \\
\hline \multirow[b]{2}{*}{7125} & PSQ & 20 & 22 & 15 & 11 & 12 & & 16 & \multirow[b]{2}{*}{347} & \multirow[b]{2}{*}{1190} \\
\hline & Thera & & & 10 & 9 & 12 & 11 & 10 & & \\
\hline \multirow[b]{2}{*}{4109} & PSQ & 33 & 39 & 27 & 23 & 31 & & 30 & \multirow[b]{2}{*}{1379} & \multirow[b]{2}{*}{1379} \\
\hline & Thera & & & 1 & 1 & 1 & 2 & 1 & & \\
\hline
\end{tabular}

Table 4: results for samples classified differently by PSQ and Thera assays. For patients without event occurrence, time to death or time to recurrence are written in italics. 


\section{Figure captions}

Figure 1: Comparison between Thera and PSQ. Agreement between the two tests for CpG76, CpG77, CpG78 and the mean of the 4 or 5 CpGs is described using scatter and Bland-Altman plots.

Figure 2: Kaplan-Meier analysis of overall survival (OS) according to MGMT promoter methylation status. M: patients with a value above the calculated cut-off and therefore considered as "methylated"; UM: patients with a value below or equal to the calculated cut-off and therefore considered as "unmethylated". 

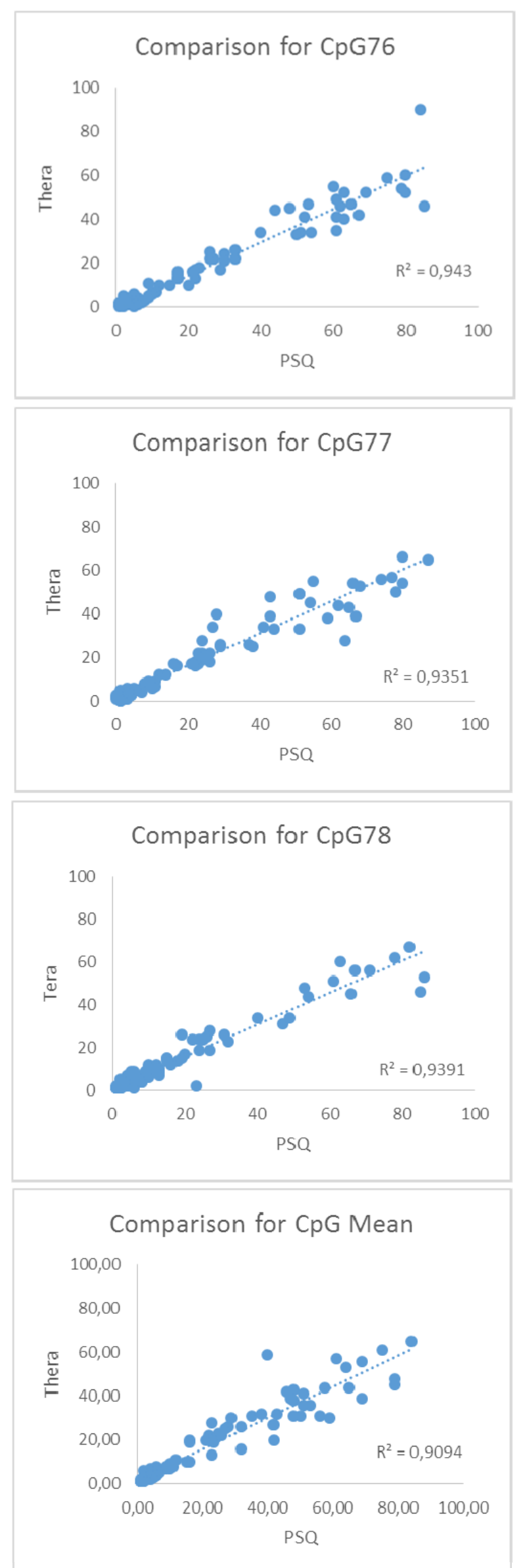
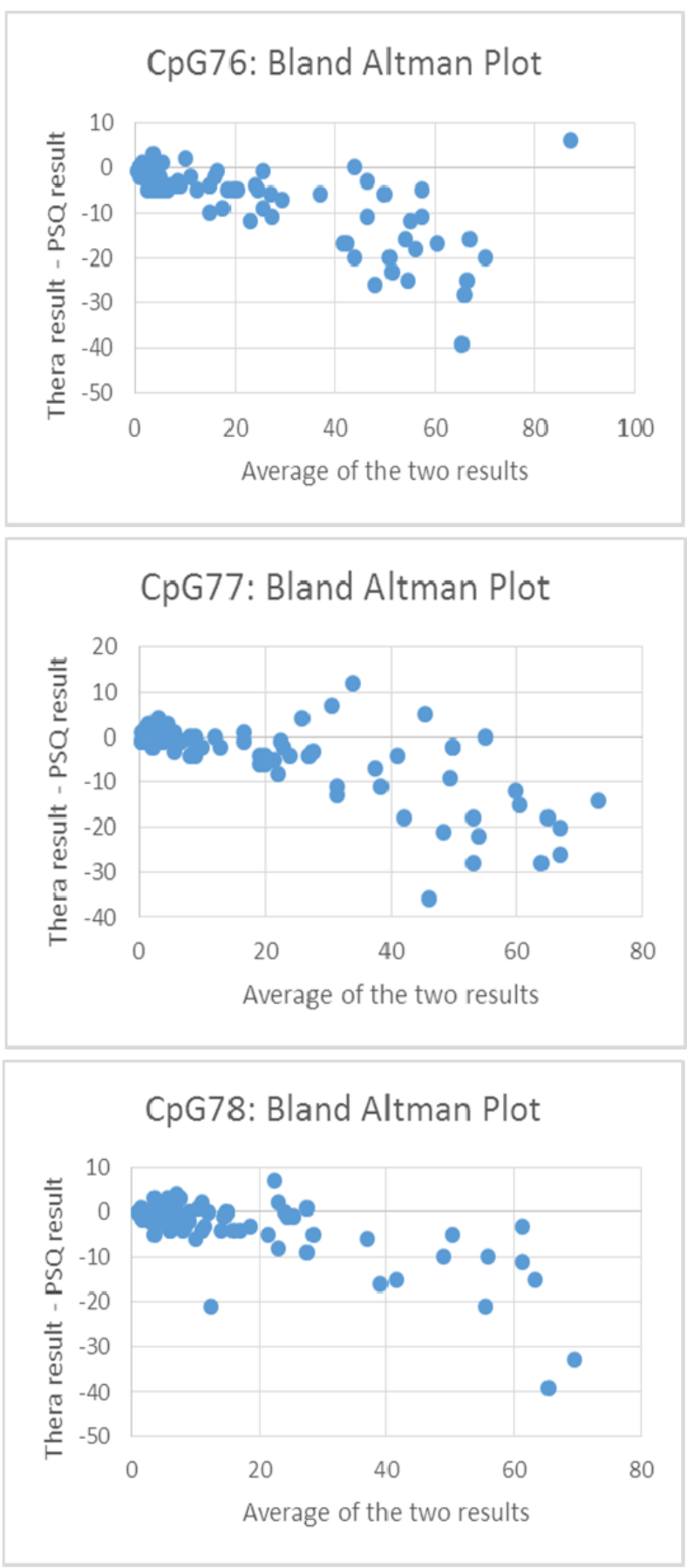

CpG mean: Bland Altman Plot

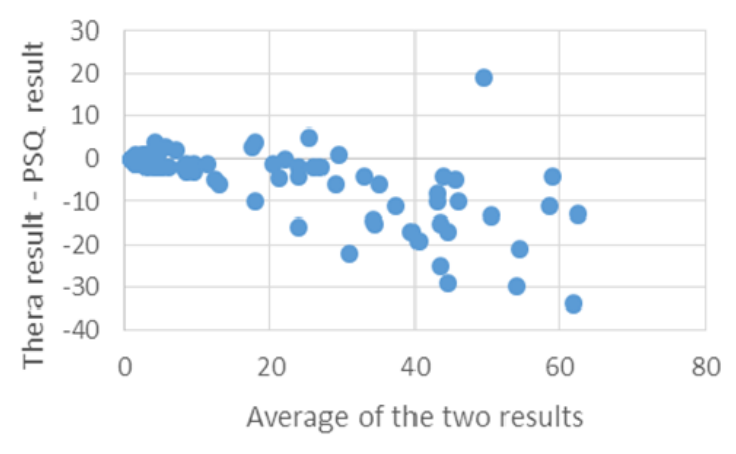

Figure 


\section{Cut-off: $8 \%$}
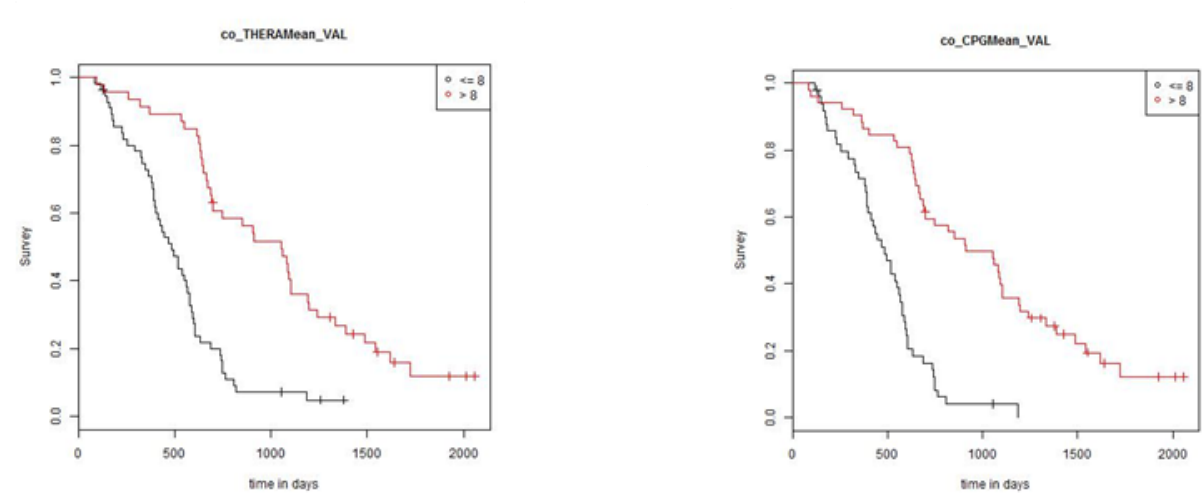

M: 34.7 (23.0-39.4)

Median time to death

UM: $15.9(13.2-19.0)$

M: 30.0 (22.9-39.1) UM: 15.9 (13.1-19.0)

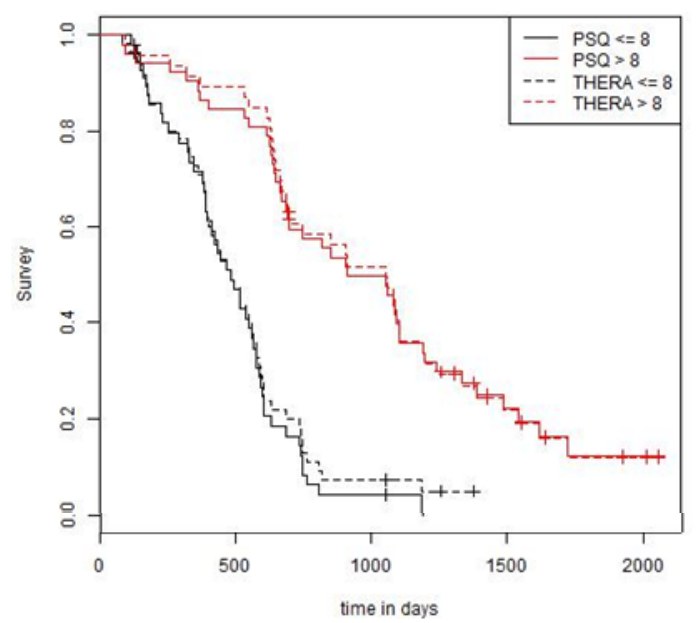

\section{Cut-off: $12 \%$}

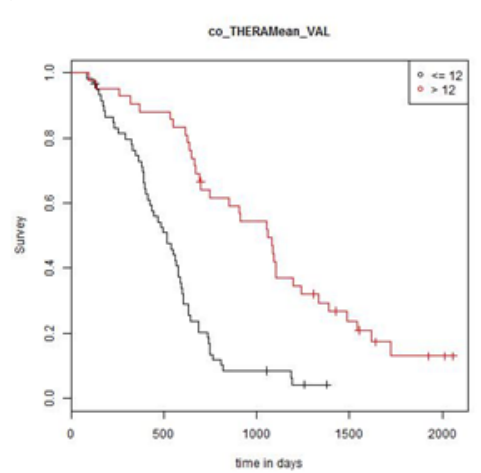

M: $34.9(24.5-43.8)$

UM: 17.0 (13.7-19.5)
Median time to death in months $(95 \% \mathrm{Cl})$

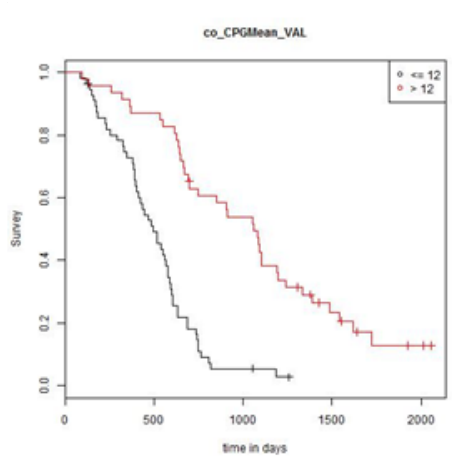

M: 34.9 (24.5-40.8) UM: 16.4 (13.7-19.1)

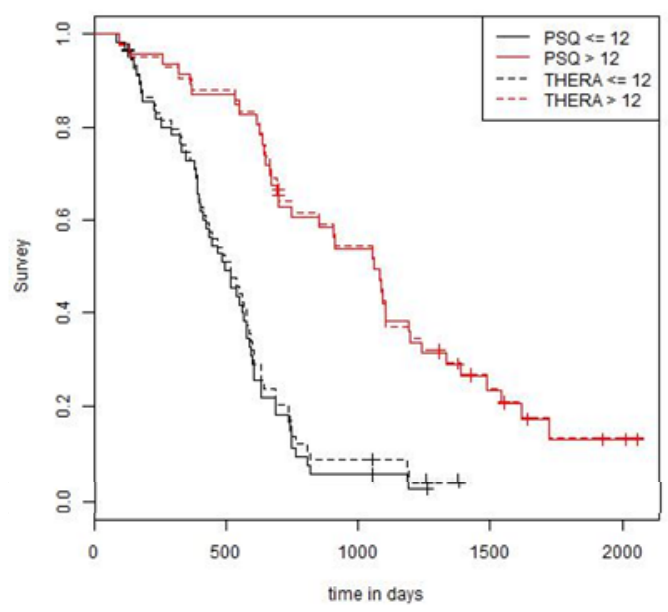


